\title{
High-purity free-electron momentum states prepared by three-dimensional optical phase modulation
}

\author{
Armin Feist $\odot,{ }^{1, *}$ Sergey V. Yalunin, ${ }^{1}$ Sascha Schäfer $\odot,{ }^{2}$ and Claus Ropers $\circledast^{1,3, \dagger}$ \\ ${ }^{1}$ IV. Physical Institute, University of Göttingen, Göttingen 37077, Germany \\ ${ }^{2}$ Institute of Physics, Carl von Ossietzky Universität Oldenburg, Oldenburg 26129, Germany \\ ${ }^{3}$ Max Planck Institute for Biophysical Chemistry (MPIBPC), Göttingen 37077, Germany
}

(Received 10 March 2020; accepted 31 August 2020; published 12 November 2020)

\begin{abstract}
We demonstrate the quantized transfer of photon energy and transverse momentum to a high-coherence electron beam. In an ultrafast transmission electron microscope, a three-dimensional phase modulation of the electron wave function is induced by transmitting the beam through a laser-illuminated thin graphite sheet. This all-optical free-electron phase space control results in high-purity superpositions of linear momentum states, providing an elementary component for optically programmable electron phase plates and beam splitters.
\end{abstract}

DOI: 10.1103/PhysRevResearch.2.043227

\section{INTRODUCTION}

The coherent manipulation of particle wave functions forms the basis of nanoscale and interference-enhanced precision measurements, ranging from matter-wave interferometry $[1,2]$ and free-electron lasers [3] to advanced electron imaging and holography [4]. In electron microscopy, phase plates and diffractive elements produce tailored quantum states, such as electron vortex beams [5-9] and self-healing Airy [10] or nondiffractive Bessel beams [11-13], with prospects for nanoscale chiral sensing $[14,15]$ and manipulations $[16,17]$.

Currently, optical field control of electron beams is experiencing growing interest from an applied and fundamental perspective [18-25]. Recently, a Zernicke phase plate based on the ponderomotive potential in optical fields was demonstrated [26]. Furthermore, high-intensity laser pulses in free space or enhanced by cavities, waveguides, and nanostructures facilitate electron pulse characterization [27-31], compression [32,33], acceleration [34,35], and attosecond structuring [36-38]. Electron phase-space manipulation within the cycle of the electromagnetic field is accessible in the terahertz [39-47] and radio-frequency domains [48-52].

Ultrafast transmission electron microscopy (UTEM) [53-55], an emerging approach to study nanoscale dynamics, is particularly suited for the study of coherent electron-light interactions, including near-field mediated inelastic scattering [56-59]. With UTEM, a number of experimental studies employed longitudinal momentum transfer to conduct nearfield imaging [56,59-62], characterize ultrashort electron

\footnotetext{
*Corresponding author: afeist@gwdg.de

†Corresponding author: cropers@gwdg.de

Published by the American Physical Society under the terms of the Creative Commons Attribution 4.0 International license. Further distribution of this work must maintain attribution to the author(s) and the published article's title, journal citation, and DOI.
}

pulses $[55,56,63]$, carry out plasmon excitation spectroscopy [64], and probe cavity modes [65,66]. In these experiments, electron spectroscopy revealed quantized energy transfer in multiples of the photon energy. This direct access to the longitudinal phase space enabled the observation of freeelectron Rabi oscillations [59], coherent quantum state control $[36,67,68]$, and attosecond bunching [36].

Addressing the transverse beam direction, recent experiments reported on optical deflection $[68,69]$ and streaking [31,37] and on vortex phase shaping [70] by chiral plasmonic fields [71,72]. However, the observation of quantized momentum transfer induced by visible light is challenging due to the small photon momentum and coherence limitations of photoelectron beams. To date, experiments on the Kapitza-Dirac effect $[73,74]$ remain the only demonstrations of a quantized transverse electron beam deflection by optical fields which leads to individual, angularly separated beams.

Here, we report the optical preparation and observation of high-purity quantum coherent linear momentum superposition states in the transverse and longitudinal directions of an electron microscope beam. Low-emittance ultrashort electron pulses, generated by nanotip photoemission, are collimated to a micrometer-scale transverse coherence length and transmitted through a laser-illuminated graphite thin film. The imprinted three-dimensional sinusoidal phase modulation yields a coherent superposition of correlated energymomentum ladder states, which is mapped by its far-field scattering distribution.

\section{THEORETICAL DESCRIPTION}

We begin by considering the theoretical basis for our study. Inelastic electron-light scattering is well-understood, and several approaches have been presented to derive analytical expressions for the photon sideband amplitudes, including a Green's function formalism [58], direct integration of the Schrödinger equation [57], and a ladder operator formalism $[59,75]$. 

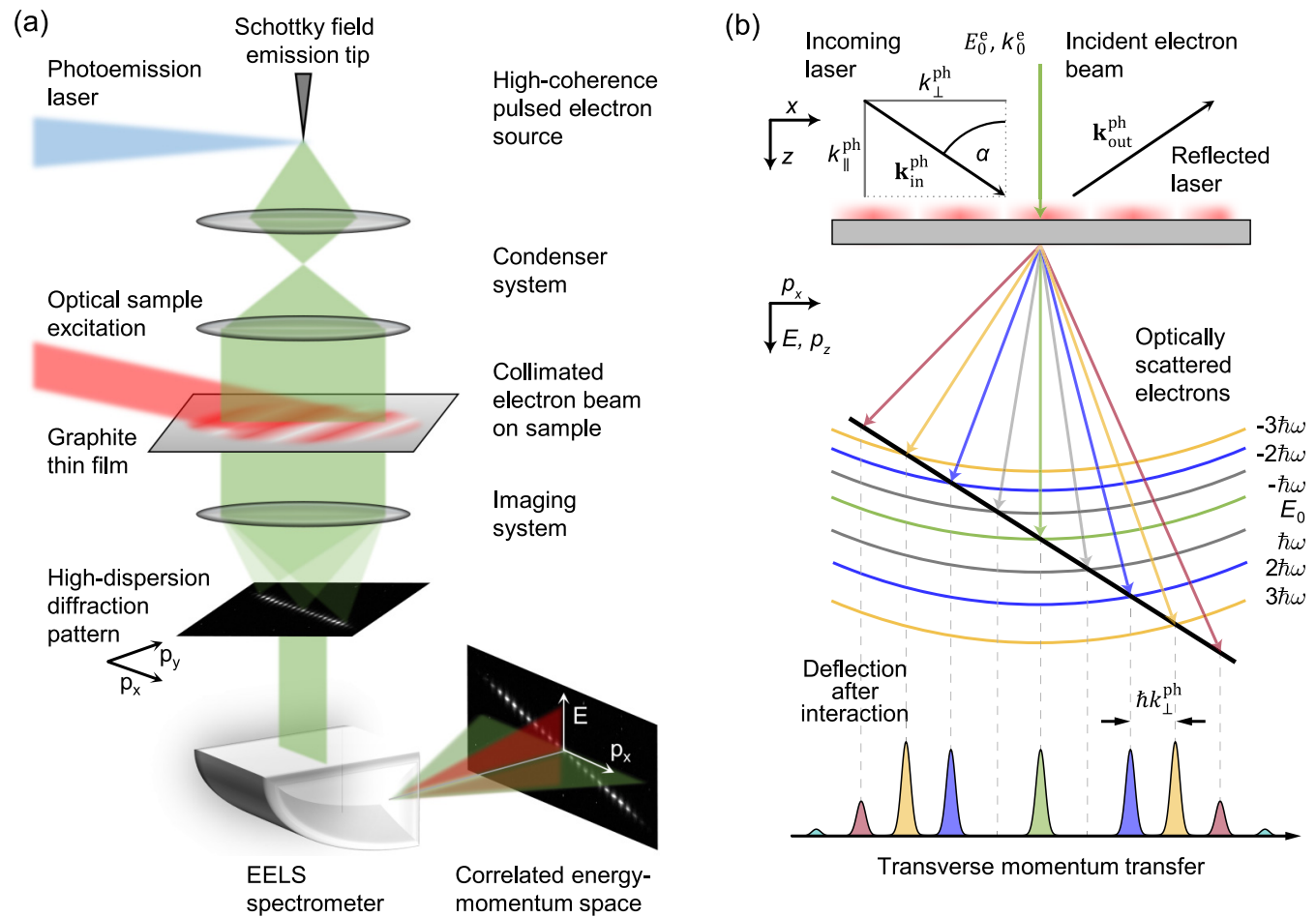

FIG. 1. Schematic setup and concept of transverse momentum resolved inelastic electron-light scattering. (a) Experimental setup. Inside of an ultrafast transmission electron microscope, photoemitted ultrashort electron pulses are collimated and transmitted through a laser sideilluminated graphite thin film. After optical interaction, the electron beam is analyzed in terms of its distributions in reciprocal (transverse) and in energy (longitudinal) space. (b) Ewald-sphere construction of inelastic electron scattering at the laser-generated traveling phase grating. The induced three-dimensional optical phase modulation yields a high-purity momentum superposition state, with separations given by integer numbers of the transverse photon momentum $\hbar k_{\perp}^{\mathrm{ph}}$ and photon energy $\hbar \omega$ (centered around the initial electron kinetic energy $\left.E_{0}^{\mathrm{e}}\right)$.

In the following, setting a general framework for transverse beam shaping, we offer an alternative, rather concise derivation using a path integral representation of the wave function [76]. In the semiclassical limit, it is expressed in terms of the classical action as $\Psi(\boldsymbol{r}, t) \sim e^{i S(\boldsymbol{r}, t) / \hbar}$, where $S(\boldsymbol{r}, t)=$ $\int_{t_{0}}^{t} L[\boldsymbol{r}(\tau)] d \tau$ is the action-a solution of the Hamilton-Jacobi equation - and $L[\boldsymbol{r}(\tau)]$ is the Lagrangian. The integral over $\tau$ is evaluated along a specific classical path $\boldsymbol{r}(\tau)$ that ends at the point $\boldsymbol{r}(t)=\boldsymbol{r}$. The initial point of the path at time $t_{0}$ is specified by the initial velocity $\dot{\boldsymbol{r}}\left(t_{0}\right)=\boldsymbol{v}=c^{2} \boldsymbol{p} / E(\boldsymbol{p})$. Consequently, the phase difference $\Phi(\boldsymbol{r}, t)$ between the phases of the initial plane-wave state and the final state at position $r$ and at time $t$ can be written as

$$
\Phi(\boldsymbol{r}, t)=\frac{1}{\hbar} \int_{t_{0}}^{t}\{L[\boldsymbol{r}(\tau)]-\boldsymbol{p} \cdot \dot{\boldsymbol{r}}(\tau)-E(\boldsymbol{p})\} d \tau,
$$

where the second and third terms subtract the phase acquired by the initial state along the path of integration. Equation (1) is the exact semiclassical limit for the phase modulation. It can be further simplified under the assumption that the energy change is small compared to the initial kinetic energy of the electron. Treating the interaction as a perturbation, one obtains in the first order the following expression [77]:

$$
\Phi(\boldsymbol{r}, t)=-\frac{1}{\hbar} \int_{t_{0}}^{t} H_{I}(\boldsymbol{r}(\tau), \tau) d \tau,
$$

where $H_{I}(\boldsymbol{r}, t)=\frac{e p}{m} A_{z}(\boldsymbol{r}, t)$ is the interaction Hamiltonian (with elementary charge $e$ and electron rest mass $m$ ), and
$A_{z}(\boldsymbol{r}, t)$ is the vector potential in the propagation direction of the electron beam (which we choose along $z$ ). With the same accuracy, $\boldsymbol{r}(\tau)$ can be approximated by a straight-line path: $\boldsymbol{r}(\tau)=\boldsymbol{r}+\boldsymbol{v}(\tau-t)$, where $\boldsymbol{v}$ is the initial velocity.

The spatial and temporal structure of the phase modulation depends on the specific field geometry in a given experiment. A field distribution breaking translational invariance along the electron propagation direction, e.g., at an optical nanostructure, can induce transitions between states separated by the photon energy [56]. For translational invariance in the direction perpendicular to the beam path, such transitions will entail quantized transverse momentum transfer [78]. The simplest geometry fulfilling both requirements is given by light reflection from a planar surface $[36,37,63,68]$. This allows for a time-harmonic electromagnetic field that abruptly varies in the $z$ direction but is propagating along the $x$ axis.

Consequently, one can choose the form $A_{z}(\boldsymbol{r}, t)=$ $G(z) / \omega \sin \left(\omega t-k_{\perp}^{\mathrm{ph}} x\right)$, where $G(z)$ denotes the $z$-dependent amplitude of the electric field, with angular frequency and transverse wave-vector component $k_{\perp}^{\mathrm{ph}}$.

As a result, Eq. (2) can be concisely written in terms of the Fourier transform of $G(z)$, as follows:

$$
\begin{aligned}
\Phi(\boldsymbol{r}, t) & =\frac{e p}{m \hbar \omega} \operatorname{Im} \int_{t_{0}}^{t} G(z+v[\tau-t]) e^{i\left(k_{\perp}^{\mathrm{ph}} x-\omega \tau\right)} d \tau \\
& =2|g| \sin \left(k_{\perp}^{\mathrm{ph}} x+\omega z / v-\omega t+\arg g\right),
\end{aligned}
$$



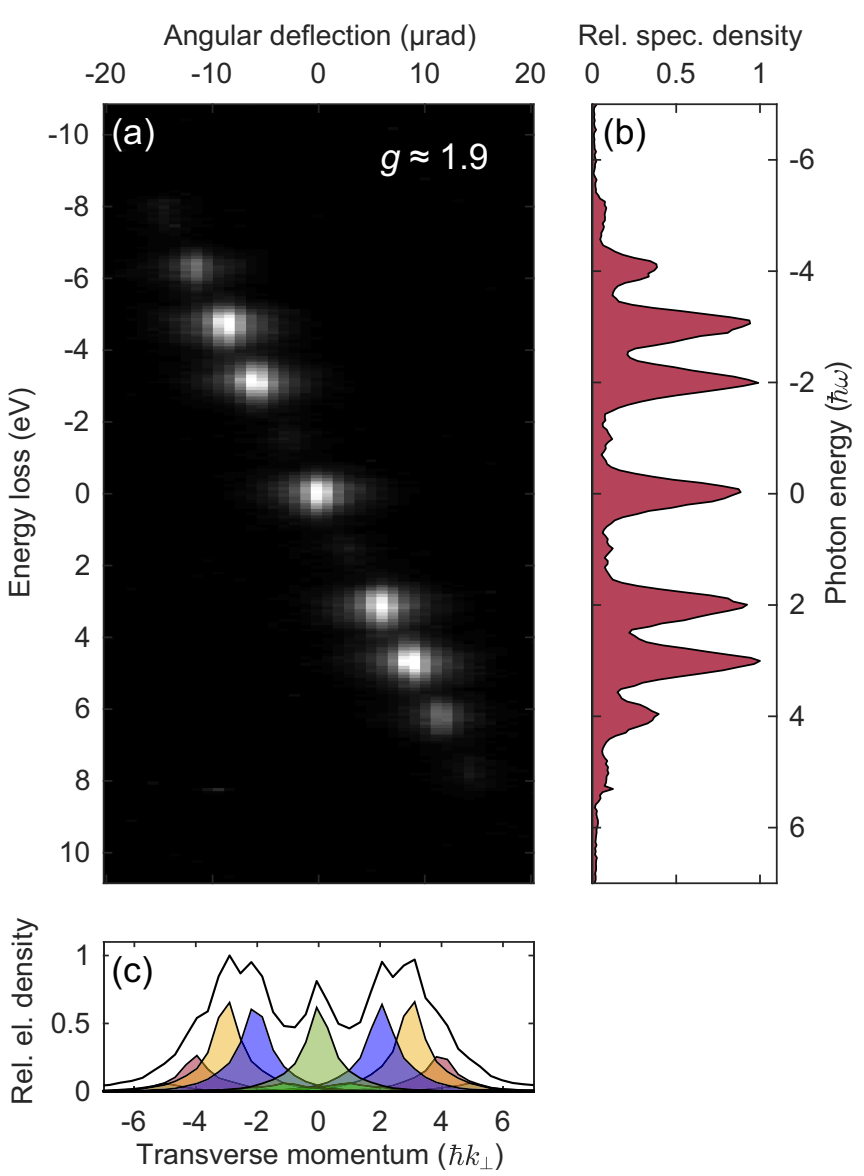

FIG. 2. Experimental energy-momentum correlation map. (a) Far-field electron scattering distribution resolved in energy and transverse momentum space (oriented along the optical plane of incidence) with normalized orthogonal projections (b) and (c) (coupling constant $g \approx 1.9$ ). (b) Spectral density as a function of the photon energy $\hbar \omega$. (c) Scattering distribution in transverse phase space with angular resolution limited by the employed electron energy loss spectrometer. The contributions of individual states are color coded.

where $g$ describes the strength of electron-light interaction:

$$
g=\frac{e}{2 \hbar \omega} \int_{-\infty}^{\infty} G(z) e^{-i \omega z / v} d z .
$$

It is convenient to deal with the phase modulation in a shifted frame [given by the substitution $z-v t \rightarrow z$ into Eq. (3)] because, in this frame, both the phase and the initial state $\Psi_{0}(\boldsymbol{r}, t)=\exp [i(p z-E t) / \hbar]$ are independent of time $t$. Finally, we arrive at

$$
\Phi(\boldsymbol{r})=2|g| \sin \left(k_{\perp}^{\mathrm{ph}} x+\omega z / v+\arg g\right) .
$$

Electron scattering at such a time-dependent phase grating results in the formation of distinct photon orders $N$ in far-field diffraction, with amplitudes given by Bessel functions of the first kind, $P_{N}=J_{N}^{2}(2|g|)[57,59]$. Physically, the absorption of a photon with energy $\hbar \omega$ necessarily involves also absorption of its transverse momentum $p_{\perp}^{\mathrm{ph}}=\hbar k_{\perp}^{\mathrm{ph}}=h / \lambda \sin \alpha$, with wavelength $\lambda$ and angle of incidence $\alpha$ for a sample plane normal to the electron beam [cf. Fig. 1(b)]. As a consequence, a one-to-one correspondence of the scattering distribution in the total kinetic energy and the transverse momentum subspace is expected [Fig. 1(b)] [78]. We note that transverse optical phase modulation with a uniform coupling constant $g$ acts as a coherent inelastic beam splitter for free-electron beams, if the individual diffraction orders are fully separated in angle. For visible optical frequencies and near-relativistic electrons, the corresponding angular separation amounts to only a few microradians. Therefore, the experimental characterization of such phase-shaped beams, and in particular the resolution of individual scattering orders, requires electron beams of sufficiently low divergence, connected to a micrometer-scale transverse coherence length $\xi_{c}=\lambda_{\mathrm{e}} /\left(2 \pi \sigma_{\theta}\right)$, with root-meansquare (rms) angular spread $\sigma_{\theta}$ [79].

As we now demonstrate, such conditions are achieved in our ultrafast transmission electron microscope, which is based on laser-driven photoelectron emission from a Schottky field emission source $[55,80]$.

\section{EXPERIMENTAL RESULTS AND DISCUSSION}

In the experiment [Fig. 1(a)], we generate ultrashort, high-coherence electron pulses by localized single-photon photoemission from a nanoscopic, Schottky-type field emitter tip [55]. After acceleration to a kinetic energy of $200 \mathrm{keV}$ (2.51 pm de-Broglie wavelength), the electron beam is collimated to a FWHM full beam divergence of $\Delta \theta=1.0 \mu \mathrm{rad}$, resulting in a lateral coherence length of $\xi_{c}=0.93 \mu \mathrm{m}$ [cf. Fig. 3(a), top], and radially limited to a diameter of about $13 \mu \mathrm{m}$. Passing the electrons through the optical field of ultrashort laser pulses (800 $\mathrm{nm}$ center wavelength, $\sim 50 \mu \mathrm{m}$ focal spot size, up to $150 \mathrm{~nJ}$ pulse energy, dispersively stretched to $3.4 \mathrm{ps}$ duration), that are reflected from the surface of a 120-nm-thick single crystalline graphite flake $(\sim 883 \mathrm{~nm}$ effective period of the transverse optical phase grating), ${ }^{1}$ imprints a sinusoidal phase modulation onto the electron wave function in the transverse and longitudinal beam directions [Fig. 1(b)] (due to the low pulse charge of less than one electron per pulse, multielectron Coulomb effects can be excluded).

The scattered electron distribution is analyzed by operating the electron microscope in diffraction mode (effective camera length of $33 \mathrm{~m}$ ). We record maps of kinetic energy and momentum using an electron spectrometer (Fig. 2), or of the full transverse momentum distribution in the far field (Fig. 3).

Figure 2(a) shows the kinetic energy and angle-resolved far-field scattering distribution, ${ }^{2}$ clearly demonstrating the creation of an equally spaced comb of energy-momentum

\footnotetext{
${ }^{1}$ The sample is prepared by cleaving a graphite single crystal perpendicular to the $c$ axis. In the experiment, the relative angle between laser and electron beam is $\alpha=59^{\circ}$ and the sample was tilted away from the surface normal and laser incidence by $\alpha=3.5^{\circ}$, precisely determined by convergent beam electron diffraction. Notably, while other materials, e.g., thin metallic membranes, allow for analogous experiments, single crystalline graphite benefits from a low relative elastic and inelastic scattering background, a high thermal stability, and can be easily prepared in large flat areas.

${ }^{2}$ Diffraction angles calibrated with a grating replica of $463 \mathrm{~nm}$ spacing.
} 
(a)
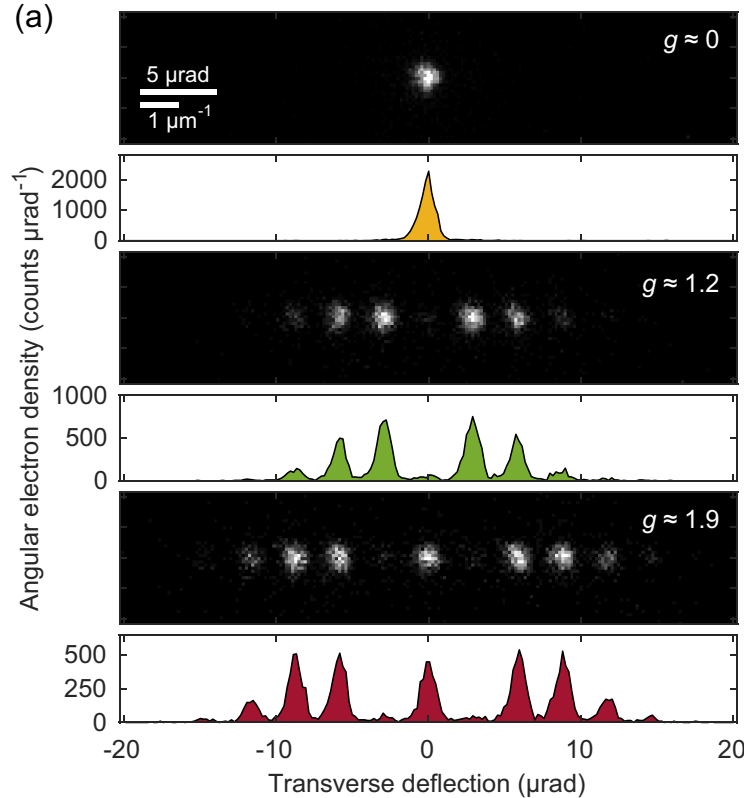

(b)

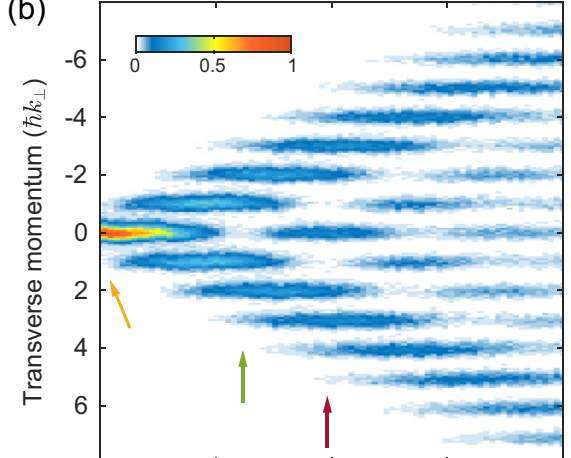

(c)

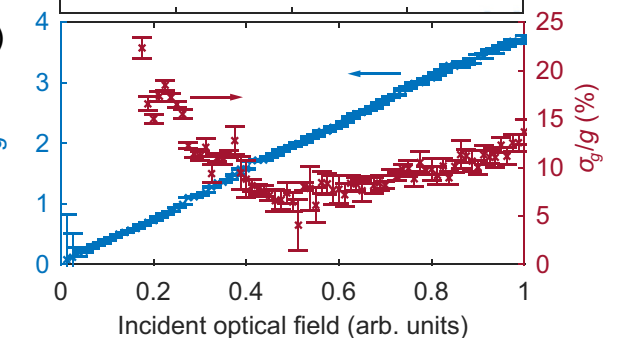

FIG. 3. Optical power-dependent far-field scattering distribution. (a) Single-electron counted high-dispersion diffraction patterns for varying optical field strength (coupling constant $g$ indicated in individual subpanels) and corresponding projection into the $k_{x}$ subspace. (b) Normalized population of transverse momentum states as a function of optical field strength. The diffraction pattern shown in (a) are indicated by arrows. (c) Extracted electron-light coupling strength, assuming a Gaussian distribution with mean $g$ (blue) and standard deviation $\sigma_{g}(\mathrm{red})$.

states [Fig. 2(b)]. The transitions in this two-dimensional energy-momentum ladder [cf. Fig. 1(b)] lead to a correlated quantized gain or loss of kinetic energy $\hbar \omega$ and transverse photon momentum $p_{\perp}^{\mathrm{ph}}=\hbar k_{\perp}^{\mathrm{ph}}$ [Fig. 2(c)], with a corresponding single-order deflection angle of $\theta_{\perp}=\arctan \left(p_{\perp}^{\mathrm{ph}} / p_{0}^{\mathrm{e}}\right)=$ $2.90 \mu \mathrm{rad}$.

Notably, while the sidebands are fully separated in energy, with a visibility of $\hbar \omega / \Delta E=2.0$ ( $\Delta E$ is the FWHM energy spread of the electron beam), the optics of the electron energy loss (EEL) spectrometer and the employed scintillatorcoupled CCD camera limit the detected angular separation to $k_{\perp}^{\mathrm{ph}} / \Delta k_{\perp}^{\mathrm{EELS}}=0.92\left(\Delta k_{\perp}^{\mathrm{EELS}}\right.$ is the FWHM of detected transverse electron momentum distribution).

Facilitated by the low-divergence electron beams used, in the following, we directly resolve individual scattering orders by their deflection angles, without employing energydispersive electron optics. We map the full two-dimensional transverse phase space by recording diffraction patterns after the optical interaction. Figure 3 shows the far-field scattering distribution using the same experimental conditions as for Fig. 2, but recording the diffracted beam by counted electron imaging with a direct detection CMOS camera (Direct Electron DE16). For increasing optical fields [cf. Fig. 3(a)], the initial single-peak transverse momentum distribution splits into an equally spaced comb, separated by the transverse photon momentum $p_{\perp}^{\mathrm{ph}}=\hbar k_{\perp}^{\mathrm{ph}}$ and well resolved with a visibility of $k_{\perp}^{\mathrm{ph}} / \Delta k_{\perp}^{\mathrm{e}}=2.9$, with ( $\Delta k_{\perp}^{\mathrm{e}}$ is the FWHM transverse momentum spread of the electron beam). The purity of the quantum superposition state prepared at a single optical interaction is encoded in the optical-field-dependent sideband populations [Fig. 3(b), top], which are, for a nonmixed state, analytically given by Bessel functions of the first kind, $J_{N}(2 g)$ [57,59]. The experimentally observed occupations are well reproduced by considering a Gaussian distributed coupling constant $g$ with standard deviation $\sigma_{g}(g)$ [Fig. 3(c)]. Beyond a value of $g=2$, we find a relative uncertainty $\sigma_{g} / g$ between $6 \%$ and $12 \%$, which indicates a high purity of the generated superposition quantum state. ${ }^{3}$ A unique fingerprint of such a state - and validation of its action as a coherent electron beam splitter-is the full suppression of specific sideband orders, e.g., in the zero-order beam $(N=0)$ for the first zero crossing of $J_{0}(2 g)$, which is achieved for $g \approx 1.2$, as is clearly visible in Fig. 3(a) (middle).

Generally, multiple sources of incoherence are of relevance: they reduce the purity of the quantum superposition state. In the current experiment, there are two main contributions, which are minimized by the design of the experiment. First, incoherent ensemble averages in the preparation of the initial quantum state (i.e., the initial spread in transverse momentum and energy) are reduced by collimating our highcoherence beam, enabling a full separation of the photon orders. Second, temporal and spatial variations in the field strength lead to averaging in $g$, which we minimize by limiting the electron beam diameter and temporally stretching the optical pulse. The simultaneous needs of a low electron beam divergence and small diameter require a picometer-scale transverse beam emittance $\varepsilon_{x, y}$. In our experiment, we achieve a value of $\varepsilon_{x, y}=\beta \gamma \sigma_{x} \sigma_{\theta}=1.63 \mathrm{pm} \mathrm{rad}$ (apertured, at the

\footnotetext{
${ }^{3}$ Lower $g$ values and a smaller number of populated states lead to a higher relative uncertainty in the fit.
} 
sample stage, with relativistic parameters $\beta$ and $\gamma$ ). Such high-brightness and low-emittance ultrashort electron pulses are readily available in field emitter-based UTEM instruments $[51,55,81,82]$.

\section{SUMMARY}

In conclusion, we have realized a coherent inelastic beam splitter for free-electron beams and demonstrate the optical preparation of high-purity transverse electron beam states.

These capabilities extend the toolset of matter-wave interferometry by providing coherent optical beam manipulation, e.g., as an element for new forms of interference-enhanced multipath electron microscopes [83]. Furthermore, taking advantage of the spectral state separation, inelastic electron holography in a scanning transmission electron microscope (STEM) type geometry [84] may give access to nonlocal information in fundamental material excitations [58,85]. Exploiting the advanced transverse phase-space control in the suboptical cycle temporal structuring of electron beams will facilitate electron microscopy with isolated attosecond electron pulses [86], reminiscent of the attosecond lighthouse in optics [87]. Finally, the concept of high-coherence transverse and longitudinal phase modulation can be extended to complex plasmonic light fields, thus, providing for an externally programmable three-dimensional all-optical phase plate for electrons.

\section{ACKNOWLEDGMENTS}

We thank the Göttingen UTEM team for ongoing support and fruitful collaboration. We acknowledge insightful discussions with Katharina E. Priebe, Tyler Harvey and Thomas Rittmann. This work was funded by the Deutsche Forschungsgemeinschaft (DFG, German Research Foundation) - 217133147/SFB 1073 (project A05), 255652344/SPP 1840 (project 'Kohärente Wechselwirkungen starker optischer Nahfelder mit freien Elektronen'), and the Gottfried Wilhelm Leibniz program.
[1] A. D. Cronin, J. Schmiedmayer, and D. E. Pritchard, Optics and interferometry with atoms and molecules, Rev. Mod. Phys. 81, 1051 (2009).

[2] F. Hasselbach, Progress in electron- and ion-interferometry, Rep. Prog. Phys. 73, 016101 (2010).

[3] C. Pellegrini, A. Marinelli, and S. Reiche, The physics of x-ray free-electron lasers, Rev. Mod. Phys. 88, 015006 (2016).

[4] P. A. Midgley and R. E. Dunin-Borkowski, Electron tomography and holography in materials science, Nat. Mater. 8, 271 (2009).

[5] M. Uchida and A. Tonomura, Generation of electron beams carrying orbital angular momentum, Nature (London) 464, 737 (2010).

[6] J. Verbeeck, H. Tian, and P. Schattschneider, Production and application of electron vortex beams, Nature (London) 467, 301 (2010).

[7] B. J. McMorran, A. Agrawal, I. M. Anderson, A. A. Herzing, H. J. Lezec, J. J. McClelland, and J. Unguris, Electron vortex beams with high quanta of orbital angular momentum, Science 331, 192 (2011).

[8] V. Grillo, G. Carlo Gazzadi, E. Karimi, E. Mafakheri, R. W. Boyd, and S. Frabboni, Highly efficient electron vortex beams generated by nanofabricated phase holograms, Appl. Phys. Lett. 104, 043109 (2014).

[9] K. Y. Bliokh, I. P. Ivanov, G. Guzzinati, L. Clark, R. Van Boxem, A. Béché, R. Juchtmans, M. A. Alonso, P. Schattschneider, F. Nori, and J. Verbeeck, Theory and applications of free-electron vortex states, Phys. Rep. 690, 1 (2017).

[10] N. Voloch-Bloch, Y. Lereah, Y. Lilach, A. Gover, and A. Arie, Generation of electron Airy beams, Nature (London) 494, 331 (2013).

[11] F. Courvoisier, A. Mathis, L. Froehly, R. Giust, L. Furfaro, P. A. Lacourt, M. Jacquot, and J. M. Dudley, Sending femtosecond pulses in circles: Highly nonparaxial accelerating beams, Opt. Lett. 37, 1736 (2012).

[12] I. Kaminer, R. Bekenstein, J. Nemirovsky, and M. Segev, Nondiffracting Accelerating Wave Packets of Maxwell's Equations, Phys. Rev. Lett. 108, 163901 (2012).
[13] V. Grillo, E. Karimi, G. C. Gazzadi, S. Frabboni, M. R. Dennis, and R. W. Boyd, Generation of Nondiffracting Electron Bessel Beams, Phys. Rev. X 4, 011013 (2014).

[14] A. Asenjo-Garcia and F. J. García de Abajo, Dichroism in the Interaction between Vortex Electron Beams, Plasmons, and Molecules, Phys. Rev. Lett. 113, 066102 (2014).

[15] S. M. Lloyd, M. Babiker, G. Thirunavukkarasu, and J. Yuan, Electron vortices: Beams with orbital angular momentum, Rev. Mod. Phys. 89, 035004 (2017).

[16] J. Verbeeck, H. Tian, and G. Van Tendeloo, How to manipulate nanoparticles with an electron beam? Adv. Mater. 25, 1114 (2013).

[17] J. Verbeeck, A. Béché, K. Müller-Caspary, G. Guzzinati, M. A. Luong, and M. Den Hertog, Demonstration of a $2 \times 2$ programmable phase plate for electrons, Ultramicroscopy 190, 58 (2018).

[18] F. J. García de Abajo, Optical excitations in electron microscopy, Rev. Mod. Phys. 82, 209 (2010).

[19] E. Hemsing, G. Stupakov, D. Xiang, and A. Zholents, Beam by design: Laser manipulation of electrons in modern accelerators, Rev. Mod. Phys. 86, 897 (2014).

[20] R. J. England, R. J. Noble, K. Bane, D. H. Dowell, C.-K. Ng, J. E. Spencer, S. Tantawi, Z. Wu, R. L. Byer, E. Peralta, K. Soong, C.-M. Chang, B. Montazeri, S. J. Wolf, B. Cowan, J. Dawson, W. Gai, P. Hommelhoff, Y.-C. Huang, C. Jing, C. McGuinness, R. B. Palmer, B. Naranjo, J. Rosenzweig, G. Travish, A. Mizrahi, L. Schachter, C. Sears, G. R. Werner, and R. B. Yoder, Dielectric laser accelerators, Rev. Mod. Phys. 86, 1337 (2014).

[21] M. F. Ciappina, J. A. Pérez-Hernández, A. S. Landsman, W. A. Okell, S. Zherebtsov, B. Förg, J. Schötz, L. Seiffert, T. Fennel, T. Shaaran, T. Zimmermann, A. Chacón, R. Guichard, A. Zaïr, J. W. G. Tisch, J. P. Marangos, T. Witting, A. Braun, S. A. Maier, L. Roso, M. Krüger, P. Hommelhoff, M. F. Kling, F. Krausz, and M. Lewenstein, Attosecond physics at the nanoscale, Rep. Prog. Phys. 80, 054401 (2017). 
[22] P. Dombi, Z. Pápa, J. Vogelsang, S. V. Yalunin, M. Sivis, G. Herink, S. Schäfer, P. Groß, C. Ropers, and C. Lienau, Strongfield nano-optics, Rev. Mod. Phys. 92, 025003 (2020).

[23] N. Talebi and C. Lienau, Interference between quantum paths in coherent Kapitza-Dirac effect, New J. Phys. 21, 093016 (2019).

[24] A. Konečná and F. J. G. de Abajo, Electron Beam Aberration Correction Using Optical Near Fields, Phys. Rev. Lett. 125, 030801 (2020).

[25] O. Reinhardt and I. Kaminer, Theory of shaping electron wavepackets with light, ACS Photonics (2020), doi: 10.1021/acsphotonics.0c01133.

[26] O. Schwartz, J. J. Axelrod, S. L. Campbell, C. Turnbaugh, R. M. Glaeser, and H. Müller, Laser phase plate for transmission electron microscopy, Nature Methods 16, 1016 (2019).

[27] P. H. Bucksbaum, M. Bashkansky, and T. J. McIlrath, Scattering of Electrons by Intense Coherent Light, Phys. Rev. Lett. 58, 349 (1987).

[28] B. J. Siwick, A. A. Green, C. T. Hebeisen, and R. J. D. Miller, Characterization of ultrashort electron pulses by electron-laser pulse cross correlation, Opt. Lett. 30, 1057 (2005).

[29] C. T. Hebeisen, G. Sciaini, M. Harb, R. Ernstorfer, T. Dartigalongue, S. G. Kruglik, and R. J. D. Miller, Grating enhanced ponderomotive scattering for visualization and full characterization of femtosecond electron pulses, Opt. Express 16, 3334 (2008).

[30] M. Gao, H. Jean-Ruel, R. R. Cooney, J. Stampe, M. de Jong, M. Harb, G. Sciaini, G. Moriena, and R. J. Dwayne Miller, Full characterization of RF compressed femtosecond electron pulses using ponderomotive scattering, Opt. Express 20, 12048 (2012).

[31] M. Kozák, J. McNeur, K. J. Leedle, H. Deng, N. Schönenberger, A. Ruehl, I. Hartl, J. S. Harris, R. L. Byer, and P. Hommelhoff, Optical gating and streaking of free electrons with sub-optical cycle precision, Nat. Commun. 8, 14342 (2017).

[32] G. H. Kassier, N. Erasmus, K. Haupt, I. Boshoff, R. Siegmund, S. M. M. Coelho, and H. Schwoerer, Photo-triggered pulsed cavity compressor for bright electron bunches in ultrafast electron diffraction, Appl. Phys. B 109, 249 (2012).

[33] L. J. Wong, B. Freelon, T. Rohwer, N. Gedik, and S. G. Johnson, All-optical three-dimensional electron pulse compression, New J. Phys. 17, 013051 (2015).

[34] J. Breuer and P. Hommelhoff, Laser-Based Acceleration of Nonrelativistic Electrons at a Dielectric Structure, Phys. Rev. Lett. 111, 134803 (2013).

[35] E. A. Peralta, K. Soong, R. J. England, E. R. Colby, Z. Wu, B. Montazeri, C. McGuinness, J. McNeur, K. J. Leedle, D. Walz, E. B. Sozer, B. Cowan, B. Schwartz, G. Travish, and R. L. Byer, Demonstration of electron acceleration in a laser-driven dielectric microstructure, Nature (London) 503, 91 (2013).

[36] K. E. Priebe, C. Rathje, S. V. Yalunin, T. Hohage, A. Feist, S. Schäfer, and C. Ropers, Attosecond electron pulse trains and quantum state reconstruction in ultrafast transmission electron microscopy, Nat. Photonics 11, 793 (2017).

[37] Y. Morimoto and P. Baum, Diffraction and microscopy with attosecond electron pulse trains, Nat. Phys. 14, 252 (2018).

[38] M. Kozák, N. Schönenberger, and P. Hommelhoff, Ponderomotive Generation and Detection of Attosecond Free-Electron Pulse Trains, Phys. Rev. Lett. 120, 103203 (2018).

[39] L. Wimmer, G. Herink, D. R. Solli, S. V. Yalunin, K. E. Echternkamp, and C. Ropers, Terahertz control of nanotip photoemission, Nat. Phys. 10, 432 (2014).
[40] E. A. Nanni, W. R. Huang, K.-H. Hong, K. Ravi, A. Fallahi, G. Moriena, R. J. Dwayne Miller, and F. X. Kärtner, Terahertzdriven linear electron acceleration, Nat. Commun. 6, 8486 (2015).

[41] C. Kealhofer, W. Schneider, D. Ehberger, A. Ryabov, F. Krausz, and P. Baum, All-optical control and metrology of electron pulses, Science 352, 429 (2016).

[42] E. Curry, S. Fabbri, J. Maxson, P. Musumeci, and A. Gover, Meter-Scale Terahertz-Driven Acceleration of a Relativistic Beam, Phys. Rev. Lett. 120, 094801 (2018).

[43] D. Zhang, A. Fallahi, M. Hemmer, X. Wu, M. Fakhari, Y. Hua, H. Cankaya, A.-L. Calendron, L. E. Zapata, N. H. Matlis, and F. X. Kärtner, Segmented terahertz electron accelerator and manipulator (STEAM), Nat. Photonics 12, 336 (2018).

[44] R. K. Li, M. C. Hoffmann, E. A. Nanni, S. H. Glenzer, M. E. Kozina, A. M. Lindenberg, B. K. Ofori-Okai, A. H. Reid, X. Shen, S. P. Weathersby, J. Yang, M. Zajac, and X. J. Wang, Terahertz-based subfemtosecond metrology of relativistic electron beams, Phys. Rev. Accel. Beams 22, 012803 (2019).

[45] D. Zhang, A. Fallahi, M. Hemmer, H. Ye, M. Fakhari, Y. Hua, H. Cankaya, A.-L. Calendron, L. E. Zapata, N. H. Matlis, and F. X. Kärtner, Femtosecond phase control in high-field terahertz-driven ultrafast electron sources, Optica 6, 872 (2019).

[46] H. W. Kim, N. A. Vinokurov, I. H. Baek, K. Y. Oang, M. H. Kim, Y. C. Kim, K.-H. Jang, K. Lee, S. H. Park, S. Park, J. Shin, J. Kim, F. Rotermund, S. Cho, T. Feurer, and Y. U. Jeong, Towards jitter-free ultrafast electron diffraction technology, Nat. Photonics 14, 245 (2019).

[47] L. Zhao, Z. Wang, H. Tang, R. Wang, Y. Cheng, C. Lu, T. Jiang, P. Zhu, L. Hu, W. Song, H. Wang, J. Qiu, R. Kostin, C. Jing, S. Antipov, P. Wang, J. Qi, Y. Cheng, D. Xiang, and J. Zhang, Terahertz Oscilloscope for Recording Time Information of Ultrashort Electron Beams, Phys. Rev. Lett. 122, 144801 (2019).

[48] R. P. Chatelain, V. R. Morrison, C. Godbout, and B. J. Siwick, Ultrafast electron diffraction with radio-frequency compressed electron pulses, Appl. Phys. Lett. 101, 081901 (2012).

[49] A. Gliserin, M. Walbran, F. Krausz, and P. Baum, Sub-phononperiod compression of electron pulses for atomic diffraction, Nat. Commun. 6, 8723 (2015).

[50] J. Maxson, D. Cesar, G. Calmasini, A. Ody, P. Musumeci, and D. Alesini, Direct Measurement of Sub-10 fs Relativistic Electron Beams with Ultralow Emittance, Phys. Rev. Lett. 118, 154802 (2017).

[51] W. Verhoeven, J. F. M. van Rens, E. R. Kieft, P. H. A. Mutsaers, and O. J. Luiten, High quality ultrafast transmission electron microscopy using resonant microwave cavities, Ultramicroscopy 188, 85 (2018).

[52] C. Jing, Y. Zhu, A. Liu, K. Schliep, X. Fu, Y. Zhao, E. Montgomery, W. Rush, A. Kanareykin, M. Katz, and J. Lau, Tunable electron beam pulser for picoseconds stroboscopic microscopy in transmission electron microscopes, Ultramicroscopy 207, 112829 (2019).

[53] A. H. Zewail, 4D ultrafast electron diffraction, crystallography, and microscopy, Annu. Rev. Phys. Chem. 57, 65 (2006).

[54] D. J. Flannigan and A. H. Zewail, 4D electron microscopy: Principles and applications, Acc. Chem. Res. 45, 1828 (2012).

[55] A. Feist, N. Bach, N. Rubiano da Silva, T. Danz, M. Möller, K. E. Priebe, T. Domröse, J. G. Gatzmann, S. Rost, J. Schauss, 
S. Strauch, R. Bormann, M. Sivis, S. Schäfer, and C. Ropers, Ultrafast transmission electron microscopy using a laser-driven field emitter: Femtosecond resolution with a high coherence electron beam, Ultramicroscopy 176, 63 (2017).

[56] B. Barwick, D. J. Flannigan, and A. H. Zewail, Photoninduced near-field electron microscopy, Nature (London) 462, 902 (2009).

[57] S. T. Park, M. Lin, and A. H. Zewail, Photon-induced near-field electron microscopy (PINEM): Theoretical and experimental, New J. Phys. 12, 123028 (2010).

[58] F. J. García de Abajo, A. Asenjo-Garcia, and M. Kociak, Multiphoton absorption and emission by interaction of swift electrons with evanescent light fields, Nano Lett. 10, 1859 (2010).

[59] A. Feist, K. E. Echternkamp, J. Schauss, S. V. Yalunin, S. Schäfer, and C. Ropers, Quantum coherent optical phase modulation in an ultrafast transmission electron microscope, Nature (London) 521, 200 (2015).

[60] A. Yurtsever, R. M. van der Veen, and A. H. Zewail, Subparticle Ultrafast spectrum imaging in 4D electron microscopy, Science 335, 59 (2012).

[61] L. Piazza, T. T. A. Lummen, E. Quiñonez, Y. Murooka, B. W. Reed, B. Barwick, and F. Carbone, Simultaneous observation of the quantization and the interference pattern of a plasmonic near-field, Nat. Commun. 6, 6407 (2015).

[62] I. Madan, G. M. Vanacore, E. Pomarico, G. Berruto, R. J. Lamb, D. Mcgrouther, T. T. A. Lummen, T. Latychevskaia, F. J. García de Abajo, and F. Carbone, Holographic imaging of electromagnetic fields via electron-light quantum interference, Sci. Adv. 5, eaav8358 (2019).

[63] F. O. Kirchner, A. Gliserin, F. Krausz, and P. Baum, Laser streaking of free electrons at $25 \mathrm{keV}$, Nat. Photonics 8, 52 (2014).

[64] E. Pomarico, I. Madan, G. Berruto, G. M. Vanacore, K. Wang, I. Kaminer, F. J. García de Abajo, and F. Carbone, meV resolution in laser-assisted energy-filtered transmission electron microscopy, ACS Photonics 5, 759 (2018).

[65] O. Kfir, H. Lourenço-Martins, G. Storeck, M. Sivis, T. R. Harvey, T. J. Kippenberg, A. Feist, and C. Ropers, Controlling free electrons with optical whispering-gallery modes, Nature (London) 582, 46 (2020).

[66] K. Wang, R. Dahan, M. Shentcis, Y. Kauffmann, A. Ben Hayun, O. Reinhardt, S. Tsesses, and I. Kaminer, Coherent interaction between free electrons and a photonic cavity, Nature (London) 582, 50 (2020).

[67] K. E. Echternkamp, A. Feist, S. Schäfer, and C. Ropers, Ramsey-type phase control of free-electron beams, Nat. Phys. 12, 1000 (2016).

[68] G. M. Vanacore, I. Madan, G. Berruto, K. Wang, E. Pomarico, R. J. Lamb, D. McGrouther, I. Kaminer, B. Barwick, F. J. García de Abajo, and F. Carbone, Attosecond coherent control of free-electron wave functions using semi-infinite light fields, Nat. Commun. 9, 2694 (2018).

[69] J. Krehl, G. Guzzinati, J. Schultz, P. Potapov, D. Pohl, J. Martin, J. Verbeeck, A. Fery, B. Büchner, and A. Lubk, Spectral field mapping in plasmonic nanostructures with nanometer resolution, Nat. Commun. 9, 4207 (2018).

[70] G. M. Vanacore, G. Berruto, I. Madan, E. Pomarico, P. Biagioni, R. J. Lamb, D. McGrouther, O. Reinhardt, I. Kaminer, B. Barwick, H. Larocque, V. Grillo, E. Karimi, F. J. García de
Abajo, and F. Carbone, Ultrafast generation and control of an electron vortex beam via chiral plasmonic near fields, Nat. Mater. 18, 573 (2019).

[71] H. Kim, J. Park, S.-W. Cho, S.-Y. Lee, M. Kang, and B. Lee, Synthesis and dynamic switching of surface plasmon vortices with plasmonic vortex lens, Nano Lett. 10, 529 (2010).

[72] G. Spektor, D. Kilbane, A. K. Mahro, B. Frank, S. Ristok, L. Gal, P. Kahl, D. Podbiel, S. Mathias, H. Giessen, F. J. Meyer $\mathrm{Zu}$ Heringdorf, M. Orenstein, and M. Aeschlimann, Revealing the subfemtosecond dynamics of orbital angular momentum in nanoplasmonic vortices, Science 355, 1187 (2017).

[73] P. L. Kapitza and P. A. M. Dirac, The reflection of electrons from standing light waves, Math Proc. Cambridge Philos, Soc. 29, 297 (1933).

[74] D. L. Freimund, K. Aflatooni, and H. Batelaan, Observation of the Kapitza-Dirac effect, Nature (London) 413, 142 (2001).

[75] A. Asenjo-Garcia and F. J. García de Abajo, Plasmon electron energy-gain spectroscopy, New J. Phys. 15, 103021 (2013).

[76] L. S. Schulman, Techniques and Applications of Path Integration, edited by Schulman (Dover Publications, Mineola, NY, 2005), p. 416.

[77] A. Lubk, Paraxial quantum mechanics, in Advances in Imaging and Electron Physics (Volume 206), edited by P. W. Hawkes (Academic, London, 2018), Chap. 2, pp. 15-58.

[78] F. J. García de Abajo, B. Barwick, and F. Carbone, Electron diffraction by plasmon waves, Phys. Rev. B 94, 041404(R) (2016).

[79] T. Van Oudheusden, E. F. De Jong, S. B. van der Geer, W. P. E. M. Op 'T Root, O. J. Luiten, B. J. Siwick, W. P. E. M. Op't Root, O. J. Luiten, and B. J. Siwick, Electron source concept for single-shot sub-100 fs electron diffraction in the 100 keV range, J. Appl. Phys. 102, 093501 (2007).

[80] N. Bach, T. Domröse, A. Feist, T. Rittmann, S. Strauch, C. Ropers, and S. Schäfer, Coulomb interactions in highcoherence femtosecond electron pulses from tip emitters, Struct. Dyn. 6, 014301 (2019).

[81] F. Houdellier, G. M. Caruso, S. Weber, M. Kociak, and A. Arbouet, Development of a high brightness ultrafast transmission electron microscope based on a laser-driven cold field emission source, Ultramicroscopy 186, 128 (2018).

[82] C. Zhu, D. Zheng, H. Wang, M. Zhang, Z. Li, S. Sun, P. Xu, H Tian, Z. Li, H. Yang, and J. Li, Development of analytical ultrafast transmission electron microscopy based on laser-driven Schottky field emission, Ultramicroscopy 209, 112887 (2020).

[83] P. Kruit, R. G. Hobbs, C.-s. Kim, Y. Yang, V. R. Manfrinato, J. Hammer, S. Thomas, P. Weber, B. Klopfer, C. Kohstall, T. Juffmann, M. A. Kasevich, P. Hommelhoff, and K. K. Berggren, Designs for a quantum electron microscope, Ultramicroscopy 164, 31 (2016).

[84] F. S. Yasin, T. R. Harvey, J. J. Chess, J. S. Pierce, and B. J. McMorran, Path-separated electron interferometry in a scanning transmission electron microscope, J. Phys. D 51, 205104 (2018).

[85] H. Lichte and B. Freitag, Inelastic electron holography, Ultramicroscopy 81, 177 (2000).

[86] M. Kozák, All-Optical Scheme for Generation of Isolated Attosecond Electron Pulses, Phys. Rev. Lett. 123, 203202 (2019).

[87] K. T. Kim, C. Zhang, T. Ruchon, J.-F. Hergott, T. Auguste, D. M. Villeneuve, P. B. Corkum, and F. Quéré, Photonic streaking of attosecond pulse trains, Nat. Photonics 7, 651 (2013). 